Communications in Physics, Vol. 20, No. 4 (2010), pp. 319-324

\title{
THE CHANNEL-WIDTH DEPENDENCE OF THE LOW-TEMPERATURE HOLE MOBILITY IN Ge-RICH NARROW SQUARE QUANTUM WELL STUDIED BY THE BAND-BENDING METHOD
}

\author{
TRAN THI HAI \\ Department of Engineering and Technology, Hong Duc University \\ NGUYEN TRUNG HONG AND NGUYEN HUYEN TUNG \\ Institute of Engineering Physics, Hanoi University of Technology \\ DOAN NHAT QUANG \\ Center for Theoretical Physics, Institute of Physics, VAST
}

\begin{abstract}
We employ the theory of band-bending effects to explain the channel-width dependence of the mobility of a two-dimentional hole gas (2DHG) in narrow square $S i / S i_{1-x} G e_{x} / S i$ quantum well at high Ge content. The numerical calculation of scattering mechanisms is shown in comparison with the ones from the previous computations. Our method enables a better quantitative description of recently measured data about the dependence of the $8 \mathrm{~K}$ mobility of holes in a $S i / S i_{0.2} G e_{0.8} / S i$ quantum well on the channel width varying from $25-70 \AA$.
\end{abstract}

\section{INTRODUCTION}

The mobility of a two-dimensional hole gas (2DHG) in $p$-channel QWs is one of the most important parameters fixing its performance, however, limited by various scatterings. To improve the performance one needs to identify the key scattering mechanisms. It is well known [1] that the best way for this purpose is to study the dependence of 2DHG mobility on the experimental conditions such as sample temperature, carrier density, and well width.

Indeed, Tsujino et al. [2] have recently reported experimental date about the curve describing the evolution of the $8 \mathrm{~K}$ mobility of holes in a $p$-type $\mathrm{Si} / \mathrm{Si}_{0.2} \mathrm{Ge}_{0.8} / \mathrm{Si} \mathrm{QW}$ versus the well width varying from $L=25-70 \AA$. The authors found a noticeable decrease of its steepness from $L=45 \AA$ on. They explained the finding in terms of surface roughness scattering (for $L<45 \AA$ ) and misfit deformation potential one (for $L>45 \AA$ ), ignoring the Matthiessen's rule. This is unreasonable because the scattering rates are of the same order of magnitude. The misfit deformation potential for holes was taken in the very form for electrons, which is shown to be invalid. [3-5] The amplitude of misfit deformation potential scattering from the barrier was taken in the form of that from the well. This is not plausible because the hole distribution is mainly located in the well, decaying rapidly into the barrier. In addition, the screening of alloy disorder scattering was omitted. So, the key scattering sources for holes in the quoted system are still unclear. 
Thus, the goal of this paper is to provide a theory of the band bending effect on the low-temperature transport properties of charge carriers In Ge-rich Narrow Square Quantum Well. We develop a variational approach to the description of quantum confinement in bent-band infinite square QWs. For strained Ge channel in $\mathrm{Si}_{1-x} \mathrm{Ge}_{x} / \mathrm{Ge} / \mathrm{Si}_{1-x} \mathrm{Ge}_{x} \mathrm{QWs}$, we incorporate all possible scattering mechanisms, especially misfit deformation potential.

\section{BASIC RELATIONS}

To start with, we examine the effect from doping-induced band bending on the carrier distribution along the growth direction. The doping profile is regarded as symmetric if there are two doped layers symmetric with respect to the channel center $(z=0)$, i.e., with an equal doping density and an equal doping geometry (doping and spacer layers). With equal potential barriers the QW is a completely symmetric system. Therefore, for high enough barriers, we may take a symmetric envelop wave function for carriers (electrons or heavy holes) in the lowest subband of the QW as follows:

$$
\zeta(z)= \begin{cases}2 B \sqrt{\pi / L} \cos (\pi z / L) \cosh (c z / L), & \text { for }|z| \leq L / 2 \\ 0, & \text { for }|z|>L / 2\end{cases}
$$

with $L$ as the well width. Here, $B$ and $c$ are variational parameters to be determined. The normalization requires that

$$
\pi B^{2}\left[\gamma_{1}(c)+1\right]=1,
$$

where $\gamma_{n}(x)$ with $n$ as an integer is a simple function defined by Eq. (8) below. Thus, there is a single independent parameter, say $c$, which is a measure of the band-bending effect from double-side doping on the carrier distribution.

Within the linear transport theory, the mobility at very low temperatures are determined by the transport lifetime: $\mu=e \tau / m^{*}$, with $m^{*}$ as the in-plane effective mass of the carrier. The transport lifetime is represented in terms of the autocorrelation function $(\mathrm{ACF})$ for each disorder by $[6]$ :

$$
\frac{1}{\tau}=\frac{1}{(2 \pi)^{2} \hbar E_{F}} \int_{0}^{2 k_{F}} d q \int_{0}^{2 \pi} d \varphi \frac{q^{2}}{\left(4 k_{F}^{2}-q^{2}\right)^{1 / 2}} \frac{\left\langle|U(\mathbf{q})|^{2}\right\rangle}{\varepsilon^{2}(q)} .
$$

Here, $\mathbf{q}=(q, \varphi)$ is the $2 \mathrm{D}$ momentum transfer due to a scattering event in the $x$ - $y$ plane (in polar coordinates): $q=|\mathbf{q}|=2 k_{F} \sin (\vartheta / 2)$ with $\vartheta$ as a scattering angle. The Fermi energy is given by $E_{F}=\hbar^{2} k_{F}^{2} / 2 m^{*}$, with $k_{F}=\sqrt{2 \pi p_{s}}$ as the Fermi wave number and $p_{s}$ is the sheet density. The ACF in Eq. (3), $\left\langle|U(\mathbf{q})|^{2}\right\rangle$, is defined by an ensemble average of the 2D Fourier transform of the (unscreened) scattering potential weighted with an envelop wave function. The carriers are expected to be subject to the following scattering mechanisms: (i) alloy disorder (AD), (ii) surface roughness (SR), and (iii) misfit deformation potential (DP). The overall lifetime $\tau_{\text {tot }}$ is then determined by the ones for individual disorders according to the Matthiessen rule,

$$
\frac{1}{\tau_{\text {tot }}}=\frac{1}{\tau_{\mathrm{AD}}}+\frac{2}{\tau_{\mathrm{SR}}}+\frac{2}{\tau_{\mathrm{DP}}}
$$


where a factor of 2 is inserted to allow for equal scatterings from two doping layers and two rough interfaces. According to Eq. (3) we ought to specify the autocorrelation function in wave-vector space $\left\langle|U(\mathbf{q})|^{2}\right\rangle$ for these scattering sources.

\section{II.1. Alloy Disorder (AD)}

We begin with scattering of the 2DHG from alloy disorder located inside of the $\mathrm{Si}_{1-x} \mathrm{Ge}_{x}$ well layer. The autocorrelation function for the scattering is supplied in the familiar form:

$$
\left\langle\left|U_{\mathrm{AD}}(\mathbf{q})\right|^{2}\right\rangle=x(1-x) u_{\mathrm{al}}^{2} \Omega_{0} \int_{-L / 2}^{+L / 2} d z|\zeta(z)|^{4},
$$

in which $x$ is the Ge content, $u_{\text {al }}$ is the alloy potential, and $L$ is, as before, the $\operatorname{Si}_{1-x} \mathrm{Ge}_{x}$ well width. The volume occupied by one alloy atom is given by $\Omega_{0}=a^{3}(x) / 8$, with $a(x)$ the lattice constant of the alloy. By means of Eq. (1) for the lowest-subband wave function, this is rewritten in terms of the variational parameter $c$ as follows:

$$
\begin{array}{r}
\left\langle\left|U_{\mathrm{AD}}(\mathbf{q})\right|^{2}\right\rangle=x(1-x) u_{\mathrm{al}}^{2} \Omega_{0} \frac{B^{4} \pi^{2}}{L} \\
\times\left[\gamma_{2}(2 c)+4 \gamma_{1}(2 c)-\gamma_{0}(2 c)\right]+4\left[\gamma_{2}(c)+4 \gamma_{1}(c)-\gamma_{0}(c)\right]+9 .
\end{array}
$$

\section{II.2. Surface Roughness (SR)}

Next, we are dealing with scattering of the 2DHG from a rough potential barrier. The scattering potential is due to roughness- induced fluctuations in the position of the barrier [7]. The autocorrelation function for surface roughness scattering in a square QW of an arbitrary depth was derived in Ref. [8]. The result reads as follows: $U_{\mathrm{SR}}(\mathbf{q})=$ $V_{0}\left|\zeta_{-}\right|^{2} \Delta_{\mathbf{q}}$, where $\Delta_{\mathbf{q}}$ is a Fourier transform of the roughness profile.

$$
\begin{array}{r}
V_{0}\left|\zeta_{-}\right|^{2}=\left[E(c)-V_{H}(0)\right] \zeta^{2}(0)+\frac{\pi^{3} e^{2} B^{4} p_{s}}{2 \varepsilon_{L}}\left\{\frac{1}{c^{2}+\pi^{2}}\right. \\
\times\left[\frac{2 c^{2}+\pi^{2}}{c}\left[\theta_{1}(2 c)+2 \theta_{1}(c)\right]+\frac{c}{2}\left[\theta_{2}(2 c)+2 \theta_{2}(c)\right.\right. \\
\left.-\theta_{0}(2 c)-2 \theta_{0}(c)\right]-\frac{\pi}{2}\left[\sigma_{2}(2 c)+2 \sigma_{1}(2 c)\right]-\frac{c^{2}+2 \pi^{2}}{\pi} \\
\left.\times\left[\sigma_{2}(c)+2 \sigma_{1}(c)\right]-\frac{2 c^{2}+3 \pi^{2}}{2 \pi}\left[\sigma_{2}(0)+2 \sigma_{1}(0)\right]\right] \\
\left.+2\left[\frac{\partial \theta_{1}(c)}{\partial c}+\frac{\partial \theta_{1}(0)}{\partial c}\right]\right\},
\end{array}
$$

where $E(c)$ is the total energy per particle in the lowest subband, $V_{H}(0)$ is the Hartree potential at $z=0, \varepsilon_{L}$ is the dielectric constant. Here we introduce the following auxiliary functions:

$$
\begin{gathered}
\gamma_{n}(\eta)=\left[\frac{1}{\eta}+\frac{(-1)^{n} \eta}{\eta^{2}+n^{2} \pi^{2}}\right] \sinh \eta \\
\theta_{n}(x)=\frac{\cosh x-1}{x}+\frac{x}{x^{2}+n^{2} \pi^{2}}\left[(-1)^{n} \cosh x-1\right]
\end{gathered}
$$


and

$$
\sigma_{n}(x)=\frac{n \pi}{x^{2}+n^{2} \pi^{2}}\left[(-1)^{n} \cosh x-1\right] .
$$

\section{II.3. Misfit Deformation Potential (DP)}

Lastly, interface roughness was shown $[8,9]$ to produce fluctuations in a strain field in a lattice-mismatched heterostructure. These in turn act as a scattering source on charge carriers. Further, it was proved [3-5] that the misfit deformation potentials for two kinds of carrier are quite different, viz., the one for electrons is fixed by a single normal diagonal component of the strain field, whereas the one for holes by all its components. We supply the 2D Fourier transform of the misfit DP for cubic crystals. The scattering potential associated with the top interface $(z=-L / 2)$ is given for electrons in [10]. We may obtain the ACFs for misfit DP scattering for holes in the following form:

$$
\begin{array}{r}
\left\langle\left|U_{\mathrm{DP}}^{(h)}(\mathbf{q})\right|^{2}\right\rangle=\left(\frac{\pi^{3 / 2} \alpha \epsilon_{\|} \Delta \Lambda B^{2}}{4 L}\right)^{2} \times t^{2} e^{-t}\left[\gamma_{1}(c+t / 2)\right. \\
\left.+\gamma_{1}(c-t / 2)+2 \gamma_{1}(t / 2)\right]^{2} \frac{1}{\left(1+\lambda^{2} t^{2} / 4 n\right)^{n+1}} \times\left\{\frac{3}{2}\left[b_{s}(K+1)\right]^{2}\right. \\
\left.\left(1+\sin ^{4} \varphi+\cos ^{4} \varphi\right)+\left(\frac{d_{s} G}{4 c_{44}}\right)^{2}\left(1+\sin ^{2} \varphi \cos ^{2} \varphi\right)\right\} .
\end{array}
$$

in the well $(|z| \leq L / 2)$ and zero elsewhere. In Eq. (11) $b_{s}$ and $d_{s}$ are the shear deformation potential constants of the well layer, and $\epsilon_{\|}$is the lattice mismatch specified by the Ge content and the widths of the well and barrier, and its anisotropy ratio is yielded by

$$
\alpha=2 \frac{c_{44}}{c_{11}-c_{12}},
$$

its elastic constants by

$$
K=2 \frac{c_{12}}{c_{11}}, \quad G=2\left(c_{11}+2 c_{12}\right)\left(1-\frac{c_{12}}{c_{11}}\right),
$$

with $c_{i j}$ as its elastic stiffness constants. It is clearly seen from Eq. (11) that the deformation potential related to a rough interface decays rapidly (exponentially) with an increase of the distance measured therefrom.

\section{RESULTS AND CONCLUSIONS}

We are applying our theory to explain the channel width dependence of the low $(8$ $\mathrm{K}$ )-temperature hole mobility in a narrow $\mathrm{Si} / \mathrm{Si}_{0.2} \mathrm{Ge}_{0.8} / \mathrm{Si} \mathrm{QW}$ reported in Ref. [2]. For numerical calculation, we used the lattice constants, elastic siffness constants, and shear deformation potentials for $S i$ and Ge. The barrier height depends on the Ge content $x$ as: $V_{0}=0.74 x \mathrm{eV}$. [11] The alloy potential is $u_{\mathrm{al}}=0.9 \mathrm{eV}$. [12] The out-of-plane and in-plane effective hole masses at $x=0.8$ are: $m_{z}=0.22 m_{\mathrm{e}}$, [12] and $m^{*} \sim 0.18 m_{\mathrm{e}}[2]$. The interface profile is described by a Gaussian autocorrelation function with, as x-ray reflectivity shown, [2] a roughness amplitude $\Delta=3.5 \AA$ and correlation length $\Lambda=23 \AA$.

(i) As clearly observed from Fig.1, the partial mobilities (SR and AD) in the bandbending model are smaller than the correspond ones in the flat-band model. This is caused 


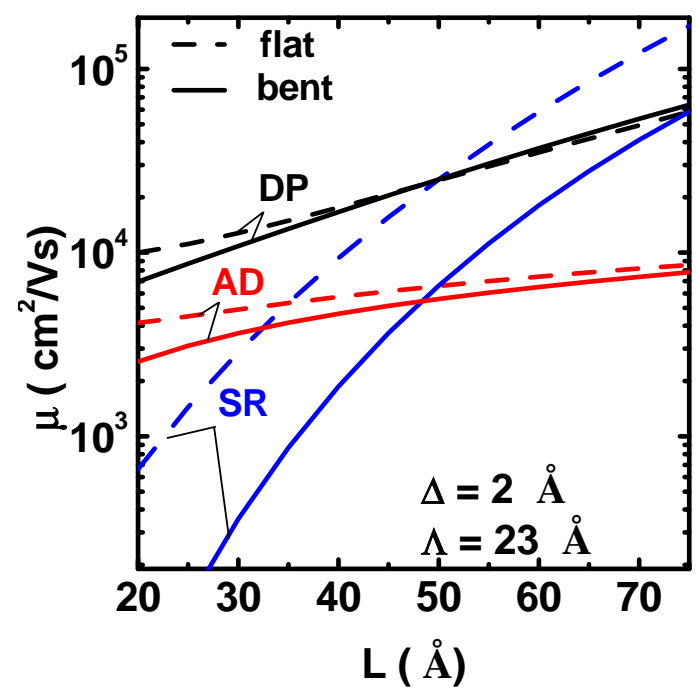

Fig. 1. The mobilities limited for $2 \mathrm{DHG}$ in a $\mathrm{Si} / \mathrm{Si}_{0.2} \mathrm{Ge}_{0.8} / \mathrm{Si} \mathrm{QW}$ vs well width $\mathrm{L}$ at a hole density $p s=1.5 * 10^{12} \mathrm{~cm}^{-2}$ in the bent-band (solid ) and flat-band (dashed lines) models.

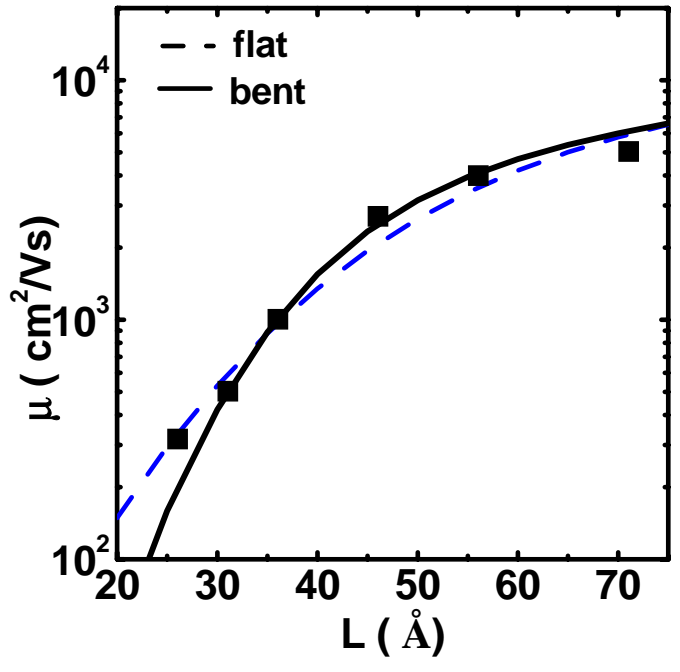

Fig. 2. Mobilities $\mu$ of holes in a $\mathrm{Si} / \mathrm{Si}_{0.2} \mathrm{Ge}_{0.8} / \mathrm{Si}$ square QW vs well width $\mathrm{L}$. The lines refers to the overall mobilities $\mu_{t o t}$ in the flat-band (dash line) and the bent-band (solid line) models. The $8 \mathrm{~K}$ experimental data [2] are marked by squares.

by the fact that the interaction between carriers in the infinite QW (Bent-band model) is larger than the one in the finite QW (flat-band model).

(ii) An inspection of Fig. 2 indicates that the overall mobility of holes in the $\mathrm{Si} / \mathrm{Si}_{0.2} \mathrm{Ge}_{0.8} / \mathrm{Si} \mathrm{QW}$, calculated within the band-bending model of infinite QWs, reproduces very well the recent experimental data about its dependence on the channel width varying from $\mathrm{L}=25-70 \AA$. Furthermore, the results we have achieved present a better quantitative description than the previous estimations [13].

(iii) As a result, the scattering mechanisms due to surface roughness, misfit deformation potential, and alloy disorder are found to dominate the 2DHG mobility.

\section{REFERENCES}

[1] F. Schäffler, Semicond. Sci. Technol. 12 (1997) 1515.

[2] S. Tsujino, C. V. Falub, E. Müller, M. Scheinert, L. Diehl, U. Gennser, T. Fromherz, A. Borak, H. Sigg, D. Grützmacher, Y. Campidelli, O. Kermarrec, and D. Bensahel, Appl. Phys. Lett. 84 (2004) 2829.

[3] D. N. Quang, V. N. Tuoc, T. D. Huan, and P. N. Phong, Phys. Rev. B70 (2004) 195336. 
[4] G. L. Bir and G. E. Pikus, Symmetry and Strain Induced Effects in Semiconductors (Wiley, New York, 1974).

[5] C. G. Van de Walle, Phys. Rev. B39 (1989) 1871.

[6] A. Gold, Phys. Rev. B35 (1987) 723; 38 (1988) 10798.

[7] T. Ando, A. B. Fowler, and F. Stern, Rev. Mod. Phys. 54 (1982) 437.

[8] D. N. Quang, V. N. Tuoc, and T. D. Huan, Phys. Rev. B68 (2003) 195316.

[9] R. M. Feenstra and M. A. Lutz, J. Appl. Phys. 78 (1995) 6091.

[10] D. N. Quang, V. N. Tuoc, N. H. Tung, and T. D. Huan, Phys. Rev. Lett. 89 (2002) 077601; Phys. Rev. B68 (2003) 153306.

[11] A. Kahan, M. Chi, and L. Friedman, J. Appl. Phys. 75 (1994) 8012.

[12] M. V. Fischetti and S. E. Laux, J. Appl. Phys. 80 (1996) 2234.

[13] D. N. Quang, N. H. Tung, D. T. Hien, H. A. Huy, Phys. Rev. B75 (2007) 073305.

Received 15 August 2009. 